\title{
Calibrating the scientific ecosystem through meta-research.
}

Tom E. Hardwicke ${ }^{1,2 \dagger}$, Stylianos Serghiou ${ }^{2,3, \ddagger}$, Perrine Janiaud ${ }^{2, \S}$, Valentin Danchev ${ }^{2, \perp}$, Sophia Crüwell ${ }^{1,5, \#}$, Steven N. Goodman ${ }^{2,3,4, \nabla}$, John P. A. Ioannidis ${ }^{1,2,3,4,6} \|$

${ }^{1}$ Meta-Research Innovation Center Berlin (METRIC-B), QUEST Center for Transforming Biomedical Research, Berlin Institute of Health, Charité - Universitätsmedizin Berlin, Berlin, Germany.

${ }^{2}$ Meta-Research Innovation Center at Stanford (METRICS), Stanford University, California, USA.

${ }^{3}$ Department of Health Research and Policy, Stanford University, California, USA.

${ }^{4}$ Department of Medicine, Stanford University, California, USA.

${ }^{5}$ Department of Psychological Methods, University of Amsterdam, Netherlands.

${ }^{6}$ Departments of Biomedical Data Science, and of Statistics, Stanford University, California, USA.

†tom.hardwicke@charite.de ORCID: 0000-0001-9485-4952

†stelios@stanford.edu ORCID: 0000-0002-2477-6060

\$pjaniaud@stanford.edu ORCID: 0000-0001-7684-8014

${ }^{\perp}$ Vdanchev@stanford.edu ORCID: 0000-0002-7563-0168

\#sophia.cruwell@student.uva.nl ORCID: 0000-0003-4178-5820

$\nabla_{\text {steve.goodman@stanford.edu }}$

\|jioannid@stanford.edu ORCID: 0000-0003-3118-6859

Corresponding author: Tom E Hardwicke, Meta-Research Innovation Center Berlin, QUEST Center for Transforming Biomedical Research, Berlin Institute of Health, Anna-Louisa-KarschStraße 2, 10178 Berlin, Germany. tom.hardwicke@charite.de 


\begin{abstract}
Whilst some scientists study insects, molecules, brains, or clouds, other scientists study science itself. Meta-research, or "research-on-research", is a burgeoning discipline that investigates efficiency, quality, and bias in the scientific ecosystem, topics that have become especially relevant amid widespread concerns about the credibility of the scientific literature. Meta-research may help calibrate the scientific ecosystem towards higher standards by providing empirical evidence that informs the iterative generation and refinement of reform initiatives. We introduce a translational framework that involves (1) identifying problems; (2) investigating problems; (3) developing solutions; and (4) evaluating solutions. In each of these areas, we review key metaresearch endeavors and discuss several examples of prior and ongoing work. The scientific ecosystem is perpetually evolving; the discipline of meta-research presents an opportunity to use empirical evidence to guide its development and maximize its potential.
\end{abstract}

Keywords: meta-research, meta-science, methodology, bias, reproducibility, open science 


\section{INTRODUCTION}

Meta-research ("research-on-research") is a burgeoning discipline that leverages theoretical, observational, and experimental approaches to investigate quality, bias, and efficiency as research unfolds in a complex and evolving scientific ecosystem (Ioannidis et al. 2015; Ioannidis 2018). Meta-research is becoming especially relevant amid warnings of a trans-disciplinary 'credibility crisis' and concerns of suboptimal and wasteful applications of the scientific method (Chalmers \& Glasziou 2009; Ioannidis 2005; Open Science Collaboration 2015; Pashler \& Wagenmakers 2012; Baker 2016; Altman 1994; Leamer 1983). These concerns have inspired reform initiatives intended to achieve higher standards of efficiency, quality, and credibility in science (Munafò et al. 2017; Ioannidis 2014; Miguel et al. 2014; Nelson et al. 2018; Poldrack et al. 2017).

\subsection{Defining 'meta-research'}

Meta-research has been defined as "the study of research itself: its methods, reporting, reproducibility, evaluation, and incentives.” (Ioannidis 2018). Given this definition, its boundaries are broad and its thematic areas interact with several other disciplines. Scientific fields are fuzzy categories with flexible, porous, overlapping borders (Börner et al. 2012). Neighboring disciplines to meta-research include, but are not limited to, philosophy of science, history of science, sociology, research synthesis (e.g., meta-analysis), data science, journalology, bibliometrics, ethics, behavioral economics, and evidence-based medicine. All of these fields may to some extent share the goals of describing, evaluating, and improving the scientific ecosystem. 


\subsection{Historical roots}

Meta-research has deep roots in the beginnings of the scientific method when intellectuals like Francis Bacon argued for greater experimentation, openness, and collaboration (Sargent 1999). These early efforts to scrutinize and modify the scientific ecosystem were still largely based on philosophical considerations rather than systematic empirical research. Over the last century, concerns about research quality have repeatedly flared across scientific disciplines, including

psychology (Elms 1975), economics (Leamer 1983), and biomedicine (Altman 1994). In parallel, systematic investigations of topics, such as publication bias (Sterling 1959), experimenter bias (Rosenthal 1966), and statistical power (Cohen 1962), have reflected a growing shift towards empiricism: an acknowledgement that mostly theoretical arguments about research practices, methods and bias should eventually be confronted with empirical data (Faust \& Meehl 2002). Initiatives such as the Cochrane Collaboration in the domain of evidence-based medicine have achieved some success at addressing suboptimal research quality; however overall, reform efforts have often failed to gain traction. Nevertheless, the recent 'credibility crisis' has sparked a trans-disciplinary discussion (Nosek et al. 2015; Miguel et al. 2014; Chalmers \& Glasziou 2009; Poldrack et al. 2017), prompted a cascade of reform initiatives (Munafò et al. 2017; Ioannidis 2014), and catalyzed the emergence of the 'meta-research' discipline.

\subsection{A translational framework for meta-research}

We will map the endeavor of meta-research using the translational framework depicted in Figure 1. This framework is not necessarily comprehensive and aims to be descriptive rather than prescriptive. The goal is to highlight how individual meta-research projects form part of a broader effort to continuously calibrate the scientific ecosystem towards higher standards of 
efficiency, credibility, and quality. In the first stage, researchers identify potential problems in the scientific ecosystem, such as publication bias or suboptimal research design. This stage is based on conceptual development and theoretical argumentation, although occasionally modelling, simulations, or case demonstrations are employed. In the second stage, researchers conduct empirical studies to investigate the prevalence and severity of the proposed problems, often by scrutinizing the published literature or surveying working scientists. In the third stage, potential solutions are generated and implemented, such as the development of new infrastructure, policy changes by key stakeholders (e.g., universities, journals, or funders), or development of educational programs. In the final stage, researchers evaluate the effectiveness of potential solutions, either under controlled conditions or 'in the wild' as they are deployed. Ideally, later stages may also reciprocally inform earlier stages, creating feedback loops. In this review, we visit each of the stages of the framework, providing illustrative examples and discussing methodological challenges.

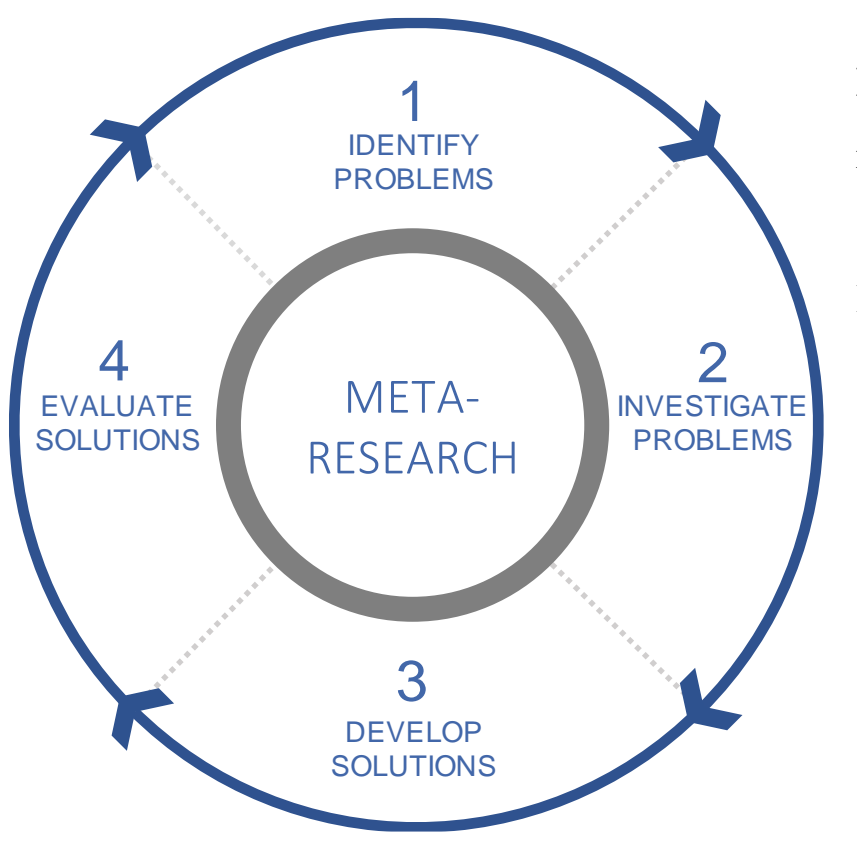

Figure 1. A translational framework for metaresearch. Figure by Hardwicke (2019) available at https://osf.io/cqrp8/ under a CC-BY4.0 license. 


\section{IDENTIFYING PROBLEMS}

Researchers may identify issues that could potentially cause inefficiency, hamper research quality, and undermine the veracity of the published literature. This can be based on theoretical arguments, modelling, simulations, or early empirical data (e.g., case demonstrations or limited evaluation of studies suffering from these problems). A central challenge is the sheer complexity of the scientific ecosystem. Multiple stakeholders, infrastructures, and processes push and pull in different directions, interact, and evolve. Any individual problem, even if it can be reasonably well described, forms part of a complex causal network of interleaved factors. Delineating symptoms vs. causes is typically not straightforward. For example, one assessment mapped 235 different biases in the biomedical literature (Chavalarias \& Ioannidis 2010) and biases may manifest differently or have different prevalence and consequences across distinct scientific fields (Fanelli et al. 2017; Goodman, 2019). Other discipline-specific characteristics, such as the ratio of true (non-null) to absent (null) relationships among those relationships under scrutiny, and design considerations, such as statistical power, and flexibility in design and analysis decisions, may translate to very different chances of getting correct or wrong answers (Ioannidis, 2005). In this section, we discuss some salient problems that have been proposed, debated, and studied.

\subsection{Incentives and norms}

Many problems may arise in the scientific ecosystem due to a fundamental misalignment of scientific ideals and incentive structures. While there is no doctrine defining a set of scientific 'ideals', the sociologist Robert Merton suggested that scientists share a set of informal cultural 'norms' (Merton 1973): Universalism (researchers should evaluate claims based on the evidence 
rather than irrelevant personal characteristics such as ethnicity, nationality, gender, or professional affiliation); Communalism (scientific methods and results belong to the entire scientific community); Disinterestedness (science should be free from personal, monetary, and other biases); and Organised Skepticism (impersonal critical scrutiny). The extent to which these norms accurately describe scientists' behaviors and beliefs has been challenged (Mulkay, 1976) although some survey evidence suggests that many working scientists subscribe to them (Anderson et al. 2010).

Several authors have asserted that key stakeholders in the scientific ecosystem are acting counter to these norms by exerting a preference for scientific findings with certain aesthetic qualities at the expense of authenticity (Nosek et al. 2012; Giner-Sorolla 2012). Specifically, stakeholders such as universities, journals, reviewers, and funders, may prefer 'newsworthy', 'positive', or 'clean' rather than 'incremental', 'negative', or 'messy' findings. Because they exert considerable influence over how research is performed and evaluated, these stakeholders could be creating selection pressures that affect the quality and veracity of research.

Several modeling and simulation efforts have explored the consequences of incentive structures by mimicking the workings of the reward system in science (Smaldino \& McElreath, 2016; Bakker et al. 2012; Grimes et al. 2018; Higginson \& Munafò, 2016). For example, Grimes et al. (2018) showed how the emphasis on 'positive' results in top-tier journals can undermine the trustworthiness of scientific findings. Conversely, trustworthiness improved when journals were agnostic as to whether a result was 'positive' or not. The authors' model also suggested that a decrease of allocated funding amplifies competition between scientists, giving rise to an environment in which false-positive results are actively rewarded, thereby further decreasing trustworthiness. Smaldino \& McElreath (2016) designed a dynamic model of competing research 
labs to demonstrate the 'natural selection of bad science'. Successful labs pursue novel, 'positive' results by selecting suboptimal methods that can deliver such results in large quantities. Ultimately these labs receive higher payoffs (e.g., citations, prestige, funding), allowing them to 'reproduce' at a higher rate and replicate through many 'offspring' labs, perpetuating suboptimal methods in the scientific ecosystem. Higginson \& Munafò (2016) extended this framework to research designs and estimated that a "publish or perish" culture contributes to designs with inadequate statistical power and high false-positive rates.

\subsection{Lack of transparency}

The importance of transparency has a long historical precedent - the world's oldest scientific institution, The Royal Society, has the motto Nullius in verba, "Take nobody's word for it". Transparency is also regarded by some as an ethical imperative. For example, the Declaration of Helsinki on ethical principles for clinical research, states that researchers have an ethical obligation to publish and disseminate complete and accurate reports of their research (World Medical Association 2013). A lack of transparency in the conduct, reporting, and dissemination of research may undermine trust in science (Vazire 2017), waste resources (Chalmers \& Glasziou 2009), and disrupt self-correction mechanisms (Ioannidis 2012).

One major concern - 'publication bias' - refers to the phenomenon whereby scientific findings are selectively published based on various aesthetic characteristics of the results, such as being 'positive' or 'newsworthy' (Dwan et al. 2013). Publication bias can emerge through multiple mechanisms. For example, it could be that journals (or reviewers) express a preference for certain types of scientific finding and selectively publish findings that best match those preferences. Alternatively/additionally, researchers may not submit findings with certain 
characteristics for publication ('file drawer' effect; Rosenthal 1979). Publication bias can also emerge from multiple sources, including selective reporting of entire studies/experiments, individual experimental conditions, specific measured outcomes, and outcomes arising from particular analyses (Phillips 2004). The consequence is that publications in academic journals fail to capture all of the findings generated by the scientific enterprise, thus providing a skewed impression of the evidentiary landscape.

Even when research findings are reported, they can be undermined by a lack of transparency about how they were generated. Much activity in the scientific ecosystem centers on research articles as a principal commodity (Young et al. 2008); however, a research article is an incomplete snapshot that cannot fully capture the rich network of research resources (e.g., protocols, materials, raw data, analysis scripts) that provide a more direct account of the research process and output (Klein et al. 2018). Being able to access research resources can facilitate independent verification, evidence synthesis, and may promote new discoveries. For example, access to raw data could enable re-analyses that probe the reproducibility and robustness of the original findings (Hardwicke et al. 2018), facilitate more sophisticated forms of meta-analysis (Tierney et al. 2015), or generate new insights through the application of novel techniques or merging with other datasets (Voytek 2016). Occasionally, overriding ethical or legal concerns may limit transparency (Meyer 2018). In such cases, explicitly declaring such 'negative constraints' on sharing should be a minimum expectation (Morey et al. 2016).

Without transparency it can be unclear what the original research hypothesis was, what the raw data looked like, how many studies/analyses were attempted, and how many unattractive results were disregarded. This information is indispensable to properly appraise research. 


\subsection{Statistical schools of thought and statistical misuse}

Most scientific claims are reinforced by a scaffold of statistical analyses that support inductive inferences from samples of data. There are multiple approaches to statistical inference, including Bayesian and likelihood-based, but frequentist inference is the most prevalent (Chavalarias et al. 2016). Deep philosophical rifts exist between these schools of thought (Mayo 2018). Frequentist inference is often used in the form of Null-Hypothesis Significance Testing (NHST), a hybrid of two different statistical schools of thought (Gigerenzer 2004; Goodman 1993) and highly prone to misinterpretation and misuse (Szucs and Ioannidis 2017b; Wagenmakers 2007).

Regardless of the statistical paradigm employed, all statistical analyses have the potential to be misused. There are many "researcher's degrees of freedom" in data analysis and interpretation - just 10 binary analytic choices result in $2^{10}=1024$ unique analysis specifications (Gelman \& Loken 2013). This flexibility can lead to large "vibration of effects" whereby many different results can be obtained from the same data and research question; results from the same study may often point in opposite directions (the so-called "Janus phenomenon"; Patel et al. 2015; Ioannidis 2008). This situation can easily be exploited, whether intentionally or not, to extract more 'desirable' results (see Section 2.1) from any given dataset.

Making analytic decisions in a data-dependent manner without using appropriate corrections generates more false-positives (Simmons et al. 2011). A series of data-dependent activities, collectively known as 'p-hacking', include stopping data collection, dropping outliers, selecting covariates, or inappropriately 'rounding' p-values based on whether those actions shift the results towards statistical significance ${ }^{1}$. Analytic flexibility varies between domains and

\footnotetext{
${ }^{1}$ To hack your own way to 'scientific glory' see https://projects.fivethirtyeight.com/p-hacking/
} 
partly depends on the degree of standardization of data processing and analysis approaches. In the field of neuroimaging for example, there is an enormous flexibility in the data processing pipeline (Carp 2012), a situation that enabled one research team to detect 'brain activity' in a dead Atlantic salmon (Bennett et al. 2009).

A related phenomenon which can interact with p-hacking, is opaque HARKing (Hypothesizing After Results are Known): presenting a finding as if it were hypothesized all along, thus encouraging false confidence in its validity (Kerr 1998; O’Boyle et al. 2013). HARKing adds further degrees of freedom to the analysis process, enabling $\mathrm{p}$-hacked findings to be explained convincingly by tailor-made hypotheses.

\subsection{Reproducibility}

Use of the term "reproducibility", and related terms like "replicability" and "repeatability", can vary across fields (Barba 2018). Goodman et al. (2016) proposed one framework which delineates "methods reproducibility" (obtaining similar results given the same data and analytical tools; often called "analytic reproducibility" or "computational reproducibility"); "results reproducibility" (obtaining similar results given the same analytical and experimental tools, but new data; often called "replication"); and "inferential reproducibility" (drawing qualitatively similar inferences from an independent methods or results reproduction of a study).

Reproducibility is a core tenet of the scientific method: if one researcher performs a study and makes a claim, a second researcher should be able to repeat the original methods and obtain similar results. Repeating original analyses with the raw data should enable recovering the originally reported findings (Hardwicke et al. 2018). However, in the context of stochastic phenomena, we should expect the findings of replication studies to differ to some extent from 
original studies (Stanley \& Spence 2014). It is also unclear how (or if) one should compare the results of two studies (an original and a replication) and conclude whether one was successful at replicating the other (Nosek \& Errington 2017; Goodman et al. 2016). Some replication attempts have sparked heated debates that often result in further non-reproducibility of inferences: despite examining the same results, researchers can disagree about what they mean (Ioannidis 2017).

\section{INVESTIGATING PROBLEMS}

At this stage, researchers conduct more in-depth empirical investigations to examine the prevalence and severity of problems. Investigations may involve meta-epidemiological assessments of potential bias, the impact of study characteristics on observed effects, the distribution of research evidence in different settings, or quantification of heterogeneity (Murad \& Wang 2017). Most studies adopt retrospective observational designs and typically involve manual examination of published research articles. A serious challenge is that low transparency may undermine efforts to systematically study other problems. Consequently, some metaepidemiological studies rely on surrogates that assess whether the pattern of published results is compatible with the theoretical impact of some particular bias. Because a number of benign factors may also contribute to such patterns, such surrogates are approximate/imperfect indicators of bias.

\subsection{Incentive structures}

In evaluations for hiring, promotion, and tenure, many institutions use simple metrics, such as the journal impact factor, which are known to be problematic (Moher et al. 2018). Survey evidence suggests that many scientists view number of publications, journal ranking, and 
authorship order as being directly associated with performance assessment and career promotions (Walker et al. 2010; van Dalen \& Henkens 2012). In some countries, including China, South Korea, and Turkey, cash rewards are offered for publishing in top-tier journals (Franzoni et al. 2011). Another strong incentive is to claim novelty, which many biomedical researchers do even when demonstrably false. In a study of 1101 randomized controlled trials with 5 or more preceding trials combined in a meta-analyses, $46 \%$ cited only 0 or 1 prior trial on the subject, a percentage that increased when there were more prior trials to cite (Robinson and Goodman, 2011). Such incentives may hinder research quality as competitive environments with greater publication pressure are more likely to report statistically significant results, a pattern which could be indicative of biased reporting (Fanelli 2010). The need to align incentive structures with good scientific practice is now widely recognized. For example, in one study about $80 \%$ of surveyed scientists thought that incentivizing better research practices would improve reproducibility (Baker 2016).

\subsection{Publication bias and selective reporting}

Empirical investigation of publication bias is challenging because unpublished studies and results are difficult to unearth. One approach is to seek out signals of publication bias in the published literature. For example, Fanelli (2011) manually examined a sample of 4656 articles across scientific disciplines and observed an overwhelming frequency of positive (i.e., "statistically significant") findings (also see Sterling 1959). Similarly, text-mining extraction of p-values from MEDLINE abstracts and full-text articles in PubMed Central showed that 96\% of abstracts and full-text articles claimed significant results with p-values $<0.05$ (Chavalarias et al. 2016). This is simply "too good to be true"; it is implausible that scientists are routinely testing 
hypotheses that so frequently turn out to be accurate, especially when statistical power is typically very low (Ioannidis \& Trikalinos 2007).

More direct evidence for publication bias has arisen from comparing public records, such as dissertations or study registries, with the published literature. For example, in the domain of management research, O'Boyle and colleagues (2013) found that the ratio of supported to unsupported hypotheses was more than twice as high in a corpus of published articles when compared to corresponding student dissertations. Similarly, Franco and colleagues (2014) capitalized on an institutional rule that required questionnaires and data underlying a series of psychology studies to be made publicly available. By comparing these materials to published studies, they observed that around $40 \%$ of studies did not report all experimental conditions, around $70 \%$ of studies did not report all outcome variables, and reported effect sizes were about twice as large as unreported effect sizes (also see Franco et al. 2016).

In medicine, researchers have used study protocols available in ethics board documentation or study registries to identify selective reporting of studies and outcomes in the published literature (Dwan et al. 2011; Chan et al. 2004; Goldacre et al. 2019; Easterbrook et al. 1991; Dickersin et al. 1992; Dechartes et al. 2017; Ross et al. 2012). For example, a systematic review of studies comparing those sources of information with their corresponding trial reports highlighted recurrent discrepancies (Dwan et al. 2014).

\subsection{Transparency of research resources}

Assessment of articles published across multiple scientific domains suggests minimal availability of critical research resources such as raw data, protocols, materials, and analysis scripts (Alsheikh-Ali et al. 2011; Hardwicke et al. 2019). Wallach et al. (2018) assessed a random sample of 149 articles published in the biomedical domain between 2015 and 2017 and found 
that 19 articles had data availability statements, 31 articles had materials availability statements, one article shared a full protocol, and no articles shared analysis scripts. Data sharing may have improved recently in some domains, but there is still much room for improvement (cf. Iqbal et al. 2016, Wallach et al. 2018).

Attempts to request research resources directly from researchers, particularly raw data, are often unsuccessful (Vines et al. 2014; Vanpaemel et al. 2015; Wicherts et al. 2006; RowhaniFarid \& Barnett 2016; Naudet et al. 2018), even for some of the most influential studies (Hardwicke \& Ioannidis 2018b). For example, Hardwicke \& Ioannidis contacted the authors of 111 highly-cited studies published in psychology and psychiatry between 2006 and 2016 and asked if they would be willing to share the associated raw data. Only 15 datasets (14\%) were made available in a completely unrestricted form and ultimately data from 76 studies (68\%) were not made available in any form. Naudet et al. were more successful and obtained 17 datasets from a sample of $37(46 \%)$ randomized controlled trials published in the BMJ and PLOS Medicine for the purpose of reanalysis.

More extensive assessment, continuous monitoring, and evaluation of research resource transparency are limited by the time-consuming nature of this type of research, which requires manual data-extraction and coding. It is possible that computational tools can be developed to automatically extract similar information, however the performance of such tools will need careful assessment to ensure reasonable sensitivity and specificity.

\subsection{Suboptimal research design}

Suboptimal research design may produce misleading results, wasting already scarce resources (Ioannidis et al. 2014). Numerous biases in research have been shown to affect the scientific 
literature (Fanelli et al. 2017) making it difficult to detect small effect sizes. Deficiencies differ from one scientific field to another, but some patterns are highly prevalent across fields. For example, lack of sufficient power to detect a range of plausible effect sizes is common across many different disciplines (Szucs \& Ioannidis 2017a; Cohen 1962; Sedlmeier \& Gigerenzer 1989; Button et al. 2013; Smaldino \& McElreath 2016; Ioannidis et al. 2017a; Moher et al. 1994). Small studies tend to generate more heterogeneous results (IntHout et al. 2015). For example, a meta-epidemiological study assessing 85,002 forest plots from the Cochrane Database of Systematic Reviews showed that most large treatment effects originated from small studies and when aggregated with other studies, pooled treatment effects tended to be smaller (Pereira et al. 2012).

Other design considerations have also been associated with effect magnitude. For example, exaggerated treatment estimates are observed (on average) in observational studies compared to RCTs (Hemkens et al. 2016), surrogate outcomes compared to patient relevant outcomes (Ciani et al. 2013), single-center compared to multicenter clinical trials (Dechartres et al. 2011), and in studies with inadequate allocation concealment, random-sequence generation, and blinding (Page et al. 2016).

\subsection{Statistical misuse}

Intentional or unintentional misuse of statistics may occur during the selection, implementation, reporting, and interpretation of statistical analyses (Table 1). For example, a recent metaepidemiological survey found that most RCTs with subgroup claims did not adjust for multiple testing, did not use an appropriate test of interaction, and were rarely validated (Wallach et al. 2017). Similarly, a study of 157 neuroscience articles found that 79 made interaction claims but 
did not appropriately test for one (Nieuwenhuis et al. 2011); instead, they inferred interaction when the outcome in one group was statistically significant and the outcome in the other was not, a known statistical fallacy (Gelman \& Stern, 2006). The causes of statistical misuse are multifaceted. The misapplication of statistical tools may reflect a response to a flawed incentive structure (Section 2.1) or may be due to a lack of understanding and/or poor training, leading to repeated use of 'mindless' statistical rituals (Gigerenzer 2004).

Table 1. Illustrative cases of statistical misuse affecting the selection, implementation, reporting, and interpretation of analyses reported in the published literature. Illustrative references are provided. Based on Table 1 in Hardwicke, Frank, Vazire, \& Goodman (in press).

Selection issues

- Using inappropriate statistical models (e.g., using metric models to analyze ordinal data, using an independent t-test in a repeated-measures design, neglecting model assumptions)

- Circular analysis (e.g., attempting to correlate brain activity measures with personality measures after selecting from the former only data that have surpassed a threshold; also known as "double dipping”)
Liddell \& Kruschke, 2018; Ernst \& Albers 2017; Strasak et al. 2007.

Fiedler 2011; Vul et al. 2009

\section{Implementation issues}

- Failure to account for multiplicity (e.g., multiple comparisons, optional stopping)

- Exploiting flexibility in analysis decisions in order to obtain more favorable outcomes

- Unjustified outlier exclusion (e.g., excluding data points ad-hoc in a way that makes outcomes more favorable to the hypothesis under scrutiny)

- Falsification of data
Cramer et al. 2016; John et al. 2012; Armitage et al. 1969; Wallach et al. 2017; Strasak et al. 2007.

Orben \& Przybylski 2019; Steegen et al. 2016; Carp 2012; Silberzahn et al. 2018.

Bakker \& Wicherts, 2014; John et al. 2012.

Simonsohn 2013; Fanelli, 2009; John et al. 2012; Carlisle 2012. 
- Inconsistencies (e.g., the reported combination of degrees of freedom, test statistic, and p-value are incompatible, or the reported combination of means for integer data, sample size, and number of items is incompatible)

- Misleading or suboptimal graphical presentation (e.g., inappropriate truncation of the y-axis, misidentification or non-identification or error bars, not representing distributional information)

- Incomplete or unclear design or analysis specification (e.g., not identifying statistical procedures, ambiguous description of experimental units, not reporting data exclusions)

- Incomplete reporting of outcomes (e.g., not reporting effect sizes, interval estimates, or standard deviations)

- Distorted presentation of non-significant results (e.g. spins in conclusions)

- Selective reporting (e.g., only reporting experiments, outcomes, or analyses that achieved statistical significance)

- Presenting post-hoc hypotheses as if they were specified a priori (opaque 'HARKing')
Bakker \& Wicherts, 2011; Brown \& Heathers, 2017; Nuijten et al. 2016.

Weissgerber et al. 2015; Lane and Sándor, 2009

Lazic et al. 2018; Weissgerber et al. 2018;

Hardwicke et al. 2018; Carp 2012; Vasilevsky et al. 2013; Glasziou et al. 2014; Avey et al. 2016;

Strasak et al. 2007.

Cumming et al. 2007; Counsell \& Harlow, 2017.

Boutron et al. 2010.

Franco et al. 2014, 2016; John et al. 2012; Dwan et al. 2011, 2013, 2014; Chan et al. 2004; Goldacre et al. 2019; Easterbrook et al. 1991.

Kerr, 1998; Wagenmakers et al. 2012; John et al. 2012.

\section{Interpretation issues}

- Incorrectly assuming that a non-significant outcome means that there is 'no effect'

- Assuming that the difference between significant and not significant is itself significant, or relatedly, erroneous analysis of interactions
Sedlmeier \& Gigerenzer ,1989; Schatz et al. 2005; Fidler et al. 2006; Hoekstra et al. 2006.

Nieuwenhuis et al. 2011; Wallach et al. 2017;

Gelman \& Stern, 2006. 


\subsection{Reproducibility}

Replication studies remain uncommon in many fields (Sterling 1959; Makel et al. 2012; Iqbal et al. 2016; Wallach et al. 2018; Hardwicke et al. 2019). In recent years however, a spate of highprofile replication attempts in psychology (Yong 2012; Open Science Collaboration 2015), and industry-based preclinical research (Prinz et al. 2011; Begley \& Ellis 2012), have generated serious concern about a trans-disciplinary "reproducibility crisis" (Baker 2016). A series of multi-laboratory efforts adopting high transparency standards and (typically) relatively large sample sizes have been deployed to investigate replicability across several fields. The Reproducibility Project: Psychology (RPP) for example, set out to replicate 100 studies published in high profile journals. The project reported several indices of 'replication success'; for example, although 97 of the original studies had statistically significant results $(\mathrm{p}<.05)$, only 36 of the replications did so. When the outcome of a replication study appears to contradict previous evidence, it is important to consider several factors such as the 'track record' of the theory under scrutiny and the fidelity of the replication attempt. Some research has explored whether 'prediction markets' can be used to estimate replication success (see sidebar).

One fairly consistent pattern that has emerged from the RPP and subsequent large-scale replication studies in the social sciences is that effect sizes observed in the replication studies have been on average approximately half as large as those reported in the original studies (Camerer et al. 2016, 2018) consistent with the idea that most published effects are inflated by selective reporting and other biases (Ioannidis, 2005, 2008). A Bayesian analysis of the RPP project also highlighted that the evidential value of many of the original studies (and some of the replication studies) was too weak to support robust inferences (Etz \&Vandekerckhove 2016). 


\section{Estimating 'replication success' using prediction markets and surveys}

Prediction markets elicit group beliefs about replication success by having participants place monetary bets (Dreber et al. 2015; Camerer et al. 2018; Forsell et al. 2018; Camerer et al. 2016). Dreber et al. (2015) found that prediction markets (71\%) outperformed pre-market surveys (58\%) when participants were asked to predict 'replication success' for 44 studies from the Reproducibility Project: Psychology. However, subsequent studies evaluating social science studies observed that market beliefs were not more accurate than surveys (Camerer et al. 2016; Camerer et al. 2018). A recent study (Forsell et al. 2018) of the Many Labs 2 replication project (R. Klein et al. 2018) reported that performance depended on the type of replication outcomes being predicted. Prediction markets more accurately predicted replication significance (i.e., a statistically significant effect in the same direction as the original study) whereas surveys more accurately predicted replication effect sizes. The relatively low cost and rapid results of prediction markets and surveys make them attractive tools for predicting replication outcomes. However, in existing assessments, forecasters had prior information about the studies under scruntiny. Thus, a future challenge for market predictions will be their performance with new, unfamiliar studies.

Several studies have also investigated whether published findings can be recovered by repeating the original analysis on the raw data (i.e., "Analytic reproducibility"; e.g., Stodden et al. 2018; Naudet et al. 2018; Hardwicke et al. 2018). For example, Hardwicke et al. encountered at least one non-reproducible value in 24 out of 35 published psychology articles often due to ambiguous, incomplete, or incorrect specification of the original analyses or mismanagement of data files. Some issues were resolved after the original authors provided (previously unreported) information that enabled reproducibility. Importantly, there was no clear evidence that the conclusions of the original studies had been undermined. Generally, studies of analytic reproducibility have highlighted that simple human errors are common, suggesting that greater 
attention should be paid to quality control systems and use of software tools that enable writing reproducible scientific papers (Klein et al. 2018; Marwick et al. 2017).

\section{DEVELOPING SOLUTIONS}

During this stage researchers and other stakeholders such as universities, funders, and journals, attempt to develop and implement solutions to problems delineated in previous stages in order to improve the efficiency, quality, and credibility of scientific research (Munafò et al. 2017;

Ioannidis 2014). Arguably many reform initiatives are facilitated by the availability of suitable software and technological infrastructure, such as data analysis tools that emphasize reproducibility and repositories for registering study protocols and sharing critical research resources like raw data (Spellman, 2015). Proper (re)training of the scientific workforce may also be crucial for the success of many such initiatives.

\subsection{Journal, funder, society and university policies}

Some journals, funders, academic societies, universities and other institutions, have begun to introduce policy changes trying to address problems. For example, Nosek et al. (2015) developed the Transparency and Openness Promotion (TOP) guidelines, a set of tiered policy

recommendations encompassing data sharing, materials sharing, analysis code transparency, data citation standards, design and analysis reporting, pre-registration, and replication. At the time of writing, the TOP website (https://cos.io/our-services/top-guidelines/) reports that over 5,000 organizations (including journals, publishers, and funders) are signatories, however this only involves "expressing their support of the principles of openness, transparency, and reproducibility, expressing interest in the guidelines and commit to conducting a review within a 
year of the standards and levels of adoption". The website also notes that "We know of over 1,000 journals or organizations that have implemented one or more TOP-compliant policy as of August 2018”.

\subsection{Pre-registration and Registered Reports}

Pre-registration involves creating a time-stamped, read-only copy of a study protocol (e.g., hypotheses, methods, and analysis plan) and archiving it in a registry before study commencement. The intention is to mitigate or enable detection of 'questionable research practices', such as 'p-hacking' and 'HARKing', by making clear what was planned and what was not (Nosek et al. 2018). For example, selective reporting can potentially be identified by comparison of the protocol and the report (Goldacre et al. 2019). Additionally, pre-registration may help researchers avoid 'capitalizing on chance' by exploiting (perhaps unintentionally) degrees of freedom in the analysis process. Crucially, the intention of pre-registration is not to reduce opportunities for exploratory (data-dependent) analyses, but to make clear the exploratory nature of such work (Wagenmakers et al. 2012; Kimmelman et al. 2014). Although the concept of pre-registration is emerging in the basic sciences and preclinical domains (Nosek et al. 2018), it has a longer precedent in the context of clinical trials registration (Dickersin \& Rennie 2012), and has sparked opposition and debate in some domains (e.g., cf. Lash \& Vandenbroucke 2012; Dal-Ré et al. 2014).

Implementation of pre-registration in practice depends on the research domain, the existence of legal mandates, and the registry that a researcher chooses (or is required) to use (Table 2). Some registries are tailored to the needs of specific fields and offer or require completion of specific registration templates. Templates facilitate standardization, but may not 
be optimized for some designs. Registries can also influence the level of transparency conferred by pre-registration. Some registries automatically make registrations public (e.g., ClinicalTrials.gov) and some offer an optional and time-limited embargo period during which the registration is hidden, but must eventually become public (e.g., the Open Science Framework). Others allow registrations to be kept hidden indefinitely (e.g., AsPredicted) which may help to allay concerns about ideas being 'scooped'. However, hidden registrations cannot be effectively monitored by the scientific community. Monitoring can help address issues such as creating multiple similar registrations, registering but not publishing, and registering but still using questionable research practices (Ioannidis et al. 2017b).

The "Registered Reports" article format involves embedding protocol registration directly within the publication pipeline (Chambers 2013). Study protocols are peer-reviewed and may be offered 'in principle acceptance' for publication before the study has even been conducted. By focusing on the quality of study design rather than the aesthetic appeal of the study findings, Registered Reports may improve study quality and mitigate publication bias. This type of publishing model appears to have been employed by the European Journal of Parapsychology from 1976 for almost two decades (Wiseman et al. 2019) as well as The Lancet from 1997 for at least a decade (Hardwicke \& Ioannidis 2018a). The current Registered Reports format (http://cos.io/rr) was introduced at Cortex in 2013 (Chambers 2013) and adoption has spread across journals and disciplines (Hardwicke \& Ioannidis 2018a). 
Table 2: Key characteristics of example registries.

\begin{tabular}{|c|c|c|c|c|c|c|}
\hline Registry & Field & Study type & $\begin{array}{l}\text { Mandates or } \\
\text { incentives }\end{array}$ & Template(s) & $\begin{array}{l}\text { Embargo } \\
\text { option }\end{array}$ & $\begin{array}{l}\text { Results } \\
\text { reporting }\end{array}$ \\
\hline ClinicalTrials.gov & Biomedicine & $\begin{array}{l}\text { Clinical } \\
\text { trials }\end{array}$ & $\begin{array}{l}\text { FDA regulation (legal) } \\
\text { ICMJE statement (for } \\
\text { publication) }\end{array}$ & Yes & No & $\begin{array}{l}\text { Mandatory for } \\
\text { some trials }\end{array}$ \\
\hline $\begin{array}{l}\text { American Economic } \\
\text { Association RCT } \\
\text { Registry }\end{array}$ & Economics & RCTs & $\begin{array}{l}\text { American Economic } \\
\text { Association journals } \\
\text { (for publication) }\end{array}$ & Yes & No & No \\
\hline AsPredicted & Social sciences & General & None declared & Yes & Yes & No \\
\hline $\begin{array}{l}\text { Open Science } \\
\text { Framework }\end{array}$ & All & All & $\begin{array}{l}\text { Pre-registration } \\
\text { Challenge }^{\text {a }} \text { (cash } \\
\text { incentives) }\end{array}$ & Optional & Yes & Optional \\
\hline PROSPERO & Biomedicine & $\begin{array}{l}\text { Systematic } \\
\text { reviews }\end{array}$ & $\begin{array}{l}\text { UK National Institute } \\
\text { of Health Research } \\
\text { (for funding) }\end{array}$ & Yes & No & Optional \\
\hline $\begin{array}{l}\text { WHO International } \\
\text { Clinical Trials } \\
\text { Registry Platform }^{\mathrm{a}}\end{array}$ & Biomedicine & $\begin{array}{l}\text { Clinical } \\
\text { trials }\end{array}$ & $\begin{array}{l}\text { Country specific } \\
\text { regulation (legal) } \\
\text { ICMJE statement (for } \\
\text { publication) }\end{array}$ & Mixed & Mixed & Mixed \\
\hline
\end{tabular}

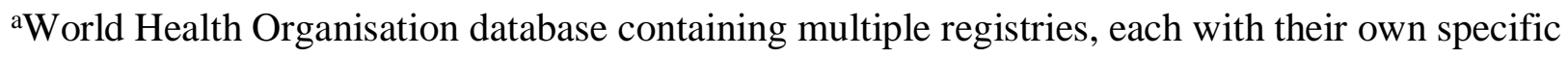
features.

\subsection{Reporting Guidelines}

Reporting guidelines are intended to draw attention to key design and analysis decisions and ensure they are adequately reported in research reports (Altman \& Simera 2016). In 2008 the EQUATOR (Enhancing the QUAlity and Transparency of health Research) network was launched to provide resources, education, and training to facilitate good research reporting (https://www.equator-network.org/). Currently the network provides 411 reporting guidelines covering a variety of study types (Table 3), but it is predominantly focused on health research. 
Table 3: Some widely used reporting guidelines available on the EQUATOR Network

\begin{tabular}{|c|c|c|c|}
\hline Study type & Guidelines & $\begin{array}{l}\text { Current } \\
\text { version }\end{array}$ & $\mathbf{N}$ citations $^{\mathrm{a}}$ \\
\hline Randomized trials & CONSORT: CONsolidated Standards Of Reporting Trials & $2010^{\mathrm{b}}$ & 6,439 \\
\hline Observational studies & $\begin{array}{l}\text { STROBE: Strengthening the Reporting of Observational Studies in } \\
\text { Epidemiology }\end{array}$ & 2008 & 8,653 \\
\hline Systematic reviews & $\begin{array}{l}\text { PRISMA: Preferred Reporting Items for Systematic Reviews and } \\
\text { Meta-Analyses }\end{array}$ & 2009 & 34,295 \\
\hline \multirow[t]{2}{*}{ Study protocols } & $\begin{array}{l}\text { SPIRIT: Standard Protocol Items: Recommendations for } \\
\text { Interventional Trials }\end{array}$ & 2013 & 997 \\
\hline & $\begin{array}{l}\text { PRISMA-P: Preferred Reporting Items for Systematic Review and } \\
\text { Meta-Analysis Protocols }\end{array}$ & 2015 & 2,524 \\
\hline \multirow{2}{*}{$\begin{array}{l}\text { Diagnostic/prognostic } \\
\text { studies }\end{array}$} & STARD: STAndards for Reporting Diagnostic accuracy studies & $2015^{b}$ & 652 \\
\hline & $\begin{array}{l}\text { TRIPOD: Transparent Reporting of a multivariable prediction } \\
\text { model for Individual Prognosis Or Diagnosis }\end{array}$ & 2015 & 821 \\
\hline Case reports & CARE: CAse REport guidelines & 2013 & 369 \\
\hline \multirow{2}{*}{$\begin{array}{l}\text { Clinical practice } \\
\text { guidelines }\end{array}$} & AGREE: Appraisal of Guidelines, Research and Evaluation & 2016 & 65 \\
\hline & RIGHT: Reporting Items for practice Guidelines in HealThcare & 2017 & 35 \\
\hline \multirow[t]{2}{*}{ Qualitative research } & SRQR: Standards for Reporting Qualitative Research & 2014 & 420 \\
\hline & COREQ: COnsolidated criteria for REporting Qualitative research & 2007 & 3,352 \\
\hline $\begin{array}{l}\text { Animal pre-clinical } \\
\text { studies }\end{array}$ & ARRIVE: Animals in Research: Reporting In Vivo Experiments & 2010 & 2,120 \\
\hline $\begin{array}{l}\text { Quality improvement } \\
\text { studies }\end{array}$ & $\begin{array}{l}\text { SQUIRE: Standards for QUality Improvement Reporting } \\
\text { Excellence }\end{array}$ & $2015^{\mathrm{b}}$ & 223 \\
\hline Economic evaluation & $\begin{array}{l}\text { CHEERS: Consolidated Health Economic Evaluation Reporting } \\
\text { Standards }\end{array}$ & 2013 & 732 \\
\hline
\end{tabular}

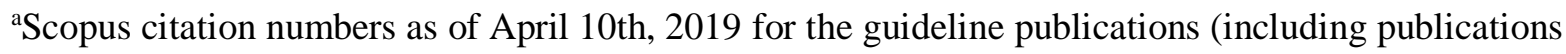
appearing simultaneously in several journals). If relevant, citation counts are shown for the latest version only.

${ }^{\mathrm{b}}$ Date refers to publication of an updated version of the guideline. 


\subsection{Peer Review}

Peer review is often considered to be an essential quality control gateway that should prevent low quality research from entering the scientific literature. There are many studies on peer review, many of which are presented at the International Conference on Peer Review that runs every four years. However, assessment of interventions to improve peer review has been relatively sparse (Bruce et al. 2016). Several variations or amendments to peer review procedures have been proposed to make it more effective and mitigate potential negative consequences, such as the introduction of bias by peer reviewers themselves. For example, there has been much debate and some empirical scrutiny of whether the identities of peer reviewers and/or the content of their reviews should be made publicly available (Ross-Hellauer \& Görögh 2019) and whether peer review procedures should be double-blind (McGillivray \& De Ranieri 2018; Justice et al. 1998). The Registered Reports publication model represents a radical departure from traditional peer review procedures, as it involves "results-blind" peer review. It has also been proposed that peer reviewers might decline to review a particular manuscript if critical research resources such as raw data, materials, and analysis scripts are not made publicly available or the manuscript does not contain a statement explaining why they cannot be made available (Morey et al. 2016). Finally, there has been discussion about whether specialized statistical review (a process which appears to have had some degree of success in biomedical domains) might also help other fields where statistical review appears to be relatively rare, such as psychology (Hardwicke, Frank, Vazire, \& Goodman, in press). 


\subsection{Collaboration}

Pooling expertise, financial, and other resources in collaborative work may improve statistical power, reproducibility, access to unique populations, and results generalizability (Ioannidis 2014; Munafò et al. 2017). Collaborative efforts have completely transformed many scientific fields, such as genetics (Seminara et al. 2007), and have become the norm in many physical and space sciences. This model has recently started to gain traction in disciplines where such largescale collaboration was previously rare, such as psychology (Open Science Collaboration, 2015; R. Klein et al. 2018). The new Psychological Science Accelerator operates as a large network of labs with different committees responsible for coordinating various tasks, such as study selection and data management (Moshontz et al. 2018). Similarly, the Observational Health Data Sciences and Informatics (OHDSI) initiative aims to bring collaborators from multiple sites together, in order to merge health data from multiple sites into one standardized database, reproduce analyses across sites, and explore the optimization of design and analytic choices in observational research (Madigan et al. 2014).

Overall, an increase in large-scale collaborations seems welcome; however, it does raise practical and conceptual issues. For example, accurately and effectively crediting hundreds of authors in a team effort could prove difficult in the current system, which values certain authorship positions more than others. Large-scale collaborations may also dilute beneficial competition and disagreement by focusing on finding a lowest common denominator consensus at the expense of more radical approaches. One interesting hybrid approach known as “adversarial collaboration" involves two (groups of) researchers who have a theoretical disagreement collaborating on a project in an effort to maximize the informational value of study design and minimize the influence of their respective biases (Matzke et al. 2015). Some 
empirical work is already addressing the relative merits of small versus large team science $(\mathrm{Wu}$ et al. 2019).

\subsection{Statistical reform}

In response to widespread statistical misuse (see Section 3.5) there have been many proposals for statistical reform (see Table 4). The American Statistician recently published a collection of 43 articles containing several such proposals (Wasserstein et al. 2019). As NHST is the dominant statistical paradigm in most disciplines (Section 2.3), many solutions are focused on either trying to improve the use of NHST or completely replace it. For example, it has been proposed that reducing the traditional threshold for declaring statistical significance from 0.05 to 0.005 would help researchers more appropriately calibrate their inferences to the strength of the evidence (Benjamin et al. 2017). It has also been suggested that, instead of using a theoretical null distribution, researchers use a data-driven null constructed using negative controls (i.e., associations that we know should be null) to produce calibrated p-values (Schuemie et al., 2014). Others have proposed completely abandoning significance testing (McShane et al. 2019). However, an empirical assessment of articles published during a journal ban of significance testing suggested a tendency to overstate conclusions (Fricker et al. 2019), which is a concern in the absence of any statistical inference (Ioannidis 2019).

Others suggest a switch to alternative paradigms, such as Bayesian statistics, which has several advantages (Wagenmakers, 2007). However, it is noteworthy that different inferential approaches often arrive at similar conclusions, at least in simple scenarios (van Dongen et al., 2019). Furthermore, the success of any given approach is largely dependent on the capabilities of the researchers conducting the statistical analyses. Statistical reform is therefore heavily dependent on resolving other issues, such as poor statistical education (Goodman, 2019; Altman, 
1994), misaligned incentives (Nosek et al., 2012), and a lack of transparency which complicates independent evaluation. Innovative approaches may be worth evaluating, such as requiring authors to indicate their degree of belief in their findings (Goodman, 2018).

\section{Table 4: Various proposed solutions for improving statistical inference on a large scale. Adapted from} Ioannidis (2018) JAMA.

\begin{tabular}{lll}
\hline Proposed solution & Application to past publications & Application to future publications \\
\hline $\begin{array}{l}\text { Lower } P \text { value } \\
\text { thresholds }\end{array}$ & A rather simple temporizing solution. & $\begin{array}{l}\text { Has potential collateral harms and success depends on } \\
\text { adoption or enforcement by stakeholders (e.g., journals, } \\
\text { funders, societies). }\end{array}$
\end{tabular}

\begin{tabular}{lll}
\hline Abandon P value & Many published P values have only & $\begin{array}{l}\text { Success depends on extent of adoption or enforcement by } \\
\text { stakeholders. }\end{array}$ \\
thresholds and instead & been reported with thresholds. &
\end{tabular}
use exact $P$ values

\begin{tabular}{lll}
\hline $\begin{array}{l}\text { Abandon P values } \\
\text { entirely }\end{array}$ & $\begin{array}{l}\text { Not easy because insufficient } \\
\text { information might be available to } \\
\text { compute other statistics; many articles } \\
\text { do not report effect sizes and/or }\end{array}$ & $\begin{array}{l}\text { Previous efforts have not gained traction. May be more } \\
\text { successful in some fields (e.g., assessment of diagnostic } \\
\text { performance or choosing predictors for prognostic models } \\
\text { in which P values would make little sense). }\end{array}$
\end{tabular}
confidence intervals.

\begin{tabular}{lll}
\hline $\begin{array}{l}\text { Focus on effect sizes } \\
\text { and their uncertainty }\end{array}$ & $\begin{array}{l}\text { Often this information is not reported at } \\
\text { all, but it has become more common in } \\
\text { recent literature. }\end{array}$ & $\begin{array}{l}\text { Relevant to the vast majority of the clinical literature, } \\
\text { should be heavily endorsed as more directly linked to } \\
\text { decision making. May be easier to promote than more } \\
\text { sophisticated solutions. }\end{array}$
\end{tabular}

(re)Train the Takes time and major commitment to Potentially a more effective solution in the long-term but scientific workforce achieve sufficient statistical literacy. may require major recasting of training priorities in curricula.

$\begin{array}{ll}\begin{array}{ll}\text { Address biases that } \\ \text { lead to inflated results }\end{array} & \begin{array}{l}\text { Requires major training; biases are } \\ \text { often impossible to detect in published } \\ \text { reports. }\end{array}\end{array}$

Pre-emptively dealing with biases is ideal. But needs concerted commitment of multiple stakeholders to promote and incentivize better research practices.

\subsection{Evidence synthesis}

The Cochrane Collaboration was founded in 1993 and quickly became a champion of evidence synthesis. Several more recent initiatives have been launched in order to encourage a more 
transparent and collaborative process for evidence synthesis such as the Stanford Metalab (http://metalab.stanford.edu/) in developmental psychology; and PROSPERO

(https://www.crd.york.ac.uk/prospero/) for pre-registration of systematic reviews. Systematic reviews and meta-analyses are growing exponentially with an increase of $2728 \%$ and $2635 \%$ respectively, between 1991 and 2014, but many are redundant, misleading, or conflicted (Ioannidis, 2016).

\section{EVALUATING SOLUTIONS}

The effectiveness of reform initiatives will depend not only on their theoretical sophistication, but on how well they are implemented. Evaluation and ongoing monitoring of reform initiatives is crucial to detect unintended negative consequences and verify that anticipated benefits are being realized in practice (Ioannidis, 2015). The success of an intervention depends on how well it is calibrated to the needs, motivations, and capability of scientists, and these factors may vary substantially across research communities. Further, any single reform initiative will typically only address a subset of a complex range of overlapping and interacting processes that are influenced by multiple stakeholders within the scientific ecosystem. Even with best intentions and flawless implementation, reform initiatives may fail because the system evolves to resist them.

Conducting informative evaluation studies can be challenging. Many initiatives are introduced without considering evaluation, which means that most evaluation studies are necessarily limited to retrospective observational designs. A given reform initiative may involve both proximal and distal goals that will emerge across the short, medium, and long term. Distal goals may take longer to materialize and be more difficult to isolate and operationalize, but they are critical indices of a reform initiative's success or failure. Proximal outcomes can be measured 
sooner, providing feedback that can be used to address weaknesses and optimize reform initiatives. Here, we describe some specific examples of evaluating solutions relevant to journal policy, reporting guidelines, pre-registration, and registered reports.

\subsection{Journal policy}

Generally, policy effectiveness appears to depend on how stringent policy requirements are, how the policy is worded and interpreted, and how robustly the policy is enforced. For example, data sharing policies that recommend authors to 'share upon request' tend to be markedly less effective relative to more stringent policies that require authors to make data publicly available in an online repository prior to publication (Rowhani-Farid \& Barnett, 2016; Nuijten et al. 2017; Hardwicke et al. 2018). For instance, Nuijten et al. observed a dramatic increase in data availability from $8.6 \%$ to $87.4 \%$ of articles after a new policy at Judgement and Decision Making asked authors to publicly share data prior to article publication. By contrast, data sharing at a comparator journal with no data sharing policy, the Journal of Behavioral Decision Making, remained negligible across the same period (pre-policy: $0 \%$; post-policy: $1.7 \%$ ).

Although some data sharing policies might achieve their proximal goal of increasing data availability, it is not necessarily the case that they are achieving their more distal goal of facilitating data re-use or results' verification. Using an interrupted time series analysis, Hardwicke et al. (2018) observed a substantial increase in data available statements after a mandatory data sharing policy was introduced at the journal Cognition (from 25\% of pre-policy articles to $78 \%$ of post-policy articles). However, amongst datasets that were reportedly available, the proportion that were actually available, complete, and understandable was only $22 \%$ pre-policy and $62 \%$ post-policy (also see Section 3.6). In response to this study, the Cognition editorial team outlined policy changes they intend to implement (Tsakiris et al. 2018) - 
an example of how meta-research can create a feedback loop between the solution evaluation and solution development stages (Figure 1).

\subsection{Reporting guidelines}

In line with ICMJE recommendations, medical journals are increasingly adopting reporting guidelines. However, an examination of the online "Instructions to authors" of 168 high impact factor journals revealed heterogenous recommendations on the use of CONSORT guidelines (Shamseer et al. 2016). Sixty-three percent endorsed the use of CONSORT of which $42 \%$ made it a prerequisite for submission. An early pre-/post- study assessing the impact of CONSORT showed improvements in completeness and transparency of published reports (Moher et al. 2001). For example, unclear description of allocation concealment significantly decreased (mean change $-22 \%$ ). Although, the overall quality of reporting improved with uptake of guidelines, it remains suboptimal and deficiencies persist (Turner et al. 2012).

\subsection{Pre-registration and Registered Reports}

In 2005, the ICMJE implemented their policy requiring trial registration to be mandatory for publication and the number of registrations on ClinicalTrials.gov increased by $73 \%$ in 6 months (Zarin et al. 2005). As of April 2019, 301,795 studies have been registered on ClinicalTrials.gov. However, many journals still publish unregistered and retrospectively registered trials (Trinquart et al. 2018; Loder et al. 2018; Gopal et al. 2018). An overview of studies evaluating the registration status of published reports in medical journals found that half of the trials published were not registered and only $20 \%$ were prospectively registered (Trinquart et al. 2018).

Registration of trials also intends to prevent selective reporting, yet changes between the registered primary outcome and published outcomes are common (Gopal et al. 2018; Scott et al. 
2015; Mathieu et al. 2009; Jones et al. 2015; Goldacre et al. 2019) with up to 31\% discrepancies in reported versus registered outcomes. Other investigators have reported that mandatory registration of primary outcomes was associated with a substantial decline in the number of trials reporting statistically significant findings, perhaps due to registration effectively mitigating selective reporting (Kaplan \& Irvin 2015).

Journal adoption of Registered Reports has many potential benefits, but a number of implementation issues were found during an exploratory investigation of the format (Hardwicke \& Ioannidis 2018a). For example, at the time of the investigation, most Registered Reports had not been formally registered and there was no reliable way of tracking their existence and status in the publication pipeline. Furthermore, most in-principle accepted protocols were not publicly available. This investigation is another example of how meta-research can create a virtuous feedback loop to inform and refine solution development (Figure 1). Many of the implementation issues identified were quickly addressed (at least in part) through the creation of a central registry for Registered Reports (http://cos.io/rr) and efforts to coordinate and update journal policy to ensure that protocols are registered and made publicly available (Chambers \& Mellor, 2018). Further evaluation and monitoring will be necessary to ascertain how effective these changes have been and if additional implementation issues should be addressed. 


\section{SUMMARY POINTS}

1. Meta-research, or "research-on-research", is a burgeoning discipline that investigates efficiency, quality, and bias in the scientific ecosystem.

2. We have introduced a translational framework that situates individual examples of meta-research within a broader research agenda which involves (1) identifying problems; (2) investigating problems; (3) developing solutions; and (4) evaluating solutions.

3. Using theoretical arguments, modelling, simulations, or early empirical data, metaresearchers have identified potential problems that might cause inefficiency, hamper research quality, and undermine the veracity of the published literature.

4. Empirical investigations have examined the prevalence and severity of problems including publication bias and selective reporting, transparency of critical research resources, suboptimal research design, incentive structures, statistical misuse, and reproducibility.

5. Scientific stakeholders such as universities, funders, journals, and researchers have introduced a number of reform initiatives related to transparency of research resources, pre-registration, Registered Reports, reporting guidelines, peer review, collaboration, statistical reform, and evidence synthesis. The goal is to improve the efficiency, quality, and credibility of scientific research.

6. Evaluation and ongoing monitoring of reform initiatives is crucial to check for unintended negative consequences and verify that anticipated benefits are being realized in practice.

7. Meta-research can help to calibrate the scientific ecosystem towards higher standards by providing a stratum of empirical evidence that informs the iterative generation and refinement of reform initiatives. 


\section{FUTURE ISSUES}

1. Meta-researchers are operating in the same scientific ecosystem as other researchers and are therefore subject to the same selection pressures that can infuse bias into the research process. It is important that meta-research is held to the same high standards expected of other research.

2. Often researchers promoting reform initiatives are also those with the interest, motivation, and means to evaluate them. These researchers may have the best of intentions, and important studies might not even be performed without their efforts, but such non-independent evaluation does create a risk of bias. Independent evaluations should be prioritized when feasible and high transparency standards are imperative.

3. As an emerging cross-disciplinary field, meta-research lacks the traditional infrastructures that support more established disciplines. Consequently, many aspects of meta-research are ad-hoc and poorly supported, including training, student recruitment, funding, and publication outlets. Career trajectories for meta-researchers are unclear as university departments specializing in meta-research are rare.

4. Meta-research is frequently complicated by a lack of transparency and poor standardization. Important information is often buried in articles and has to undergo time-consuming and error prone manual extraction. Other information is hidden in journal publishing systems or in researchers' files. Improved transparency standards combined within technological developments will enable more efficient, comprehensive, and effective meta-research.

5. Recent advances in text-mining, machine learning, and other automated tools may create new opportunities for meta-research on topics that were previously out of reach. Automated tools may also be able to enhance peer review by detecting simple errors or identifying suggestive patterns that can be evaluated further by a human.

6. Different disciplines often share similar problems but have attempted different solutions. More interdisciplinary collaboration and cross-fertilization of ideas may help to ensure that the most effective strategies are widely shared.

7. Widespread concerns about the veracity of the science literature can be unsettling, but this is an exciting time to be a scientist. The scientific method is still the best route to 
finding truths about nature and we can leverage scientific methods to study science itself. At this critical juncture, meta-research has a crucial role to play to guide scientists in their attempts to calibrate the scientific ecosystem towards higher standards of efficiency, quality, and credibility.

\section{DISCLOSURE STATEMENT}

The authors are not aware of any affiliations, memberships, funding, or financial holdings that might be perceived as affecting the objectivity of this review.

\section{ACKNOWLEDGEMENTS}

The Meta-Research Innovation Center at Stanford (METRICS) is supported by a grant from the Laura and John Arnold Foundation. The Meta-Research Innovation Center Berlin (METRIC-B) is supported by a grant from the Einstein Foundation and Stiftung Charitè (Einstein BIH Visiting Fellowship).

\section{LITERATURE CITED}

Alsheikh-Ali, AA, Qureshi W, Al-Mallah MH, Ioannidis JPA. 2011. Public availability of published research data in high-impact journals. PLOS One 6(9): e24357.

Altman DG. 1994. The scandal of poor medical research. BMJ. 308(6924): 283-84.

Altman DG, Simera I. 2016. A history of the evolution of guidelines for reporting medical research: The long road to the EQUATOR Network. J. Roy. Soc. Med. 109(2): 67-77.

Anderson MS, Ronning EA, Devries R, Martinson BC. 2010. Extending the Mertonian norms: scientists' subscription to norms of research. J. High. Ed. 81(3): 366-93.

Armitage P, McPherson CK, Rowe BC. 1969. Repeated significance tests on accumulating data. J. Roy. Stat. Soc. Series A. 132(2): 235-244. 
Avey MT, Moher D, Sullivan KJ, Fergusson D, Griffin G, Grimshaw JM, Hutton B, et al. 2016. The devil is in the details: incomplete reporting in preclinical animal research. PLOS One 11(11): e0166733.

Sargent RM. 1999. Francis Bacon: Selected Philosophical Works. Hackett Publishing. IA: USA.

Baker M. 2016. 1,500 scientists lift the lid on reproducibility. Nature. 533(7604): 452-54.

Bakker M, van Dijk A, Wicherts, JM. 2012. The rules of the game called psychological science. Perspect. Psychol. Sci. 7(6): 543-54.

Bakker M, Wicherts JM. 2011. The (mis)reporting of statistical results in psychology journals. Behav. Res. Meth. 43(3): 666-78.

Bakker M, Wicherts JM. 2014. Outlier removal, sum scores, and the inflation of the type I error rate in independent samples $t$ tests: the power of alternatives and recommendations. Psychol. Meth. 19(3): 409-427.

Barba LA. 2018. Terminologies for reproducible research. Preprint retrieved from: http://arxiv.org/abs/1802.03311.

Begley CG, Ellis LM. 2012. Raise standards for preclinical cancer research. Nature 483(7391): 531-33.

Benjamin DJ, Berger JO, Johannesson M, Nosek BA, Wagenmakers E-J, Berk R, Bollen KA, et al. 2017. Redefine statistical significance. Nat. Hum. Behav. 2(1): 6.

Bennett CM, Miller MB, Wolford GL. 2009. Neural correlates of interspecies perspective taking in the post-mortem Atlantic salmon: an argument for multiple comparisons correction. NeuroImage. 47(S1): S39-S41.

Börner K, Klavans R, Patek M, Zoss AM, Biberstine JR, Light RP, Larivière V, Boyack KW. 2012. Design and update of a classification system: the UCSD map of science." PLOS ONE. 7(7): e39464.

Boutron, I., Dutton S, Ravaud P, Altman DG. 2010. Reporting and interpretation of randomized controlled trials with statistically nonsignificant results for primary outcomes. JAMA 303(20): 2058-64.

Brown NJL, Heathers JAJ. 2017. The GRIM Test: a simple technique detects numerous anomalies in the reporting of results in psychology." Soc. Psychol. Person. Sci. 8(4): 363-69. 
Bruce R, Chauvin A, Trinquart L, Ravuad P, Boutron I. 2016. Impact of interventions to improve the quality of peer review of biomedical journals: a systematic review and meta-analysis. BMC Med. 14: 85.

Button KS, Ioannidis JPA, Mokrysz C, Nosek BA, Flint J, Robinson ESJ, Munafò MR. 2013. Power failure: Why small sample size undermines the reliability of neuroscience. Nat. Rev. Neurosci. 14(5): 365-76.

Camerer CF, Dreber A, Forsell E, Ho T-H, Huber J, Johannesson M, Kirchler M, et al. 2016. Evaluating replicability of laboratory experiments in economics. Science 351(6280): $1433-36$.

Camerer CF, Dreber A, Holzmeister F, Ho T-H, Huber J, Johannesson M, Kirchler M, et al. 2018. Evaluating the replicability of social science experiments in nature and science between 2010 and 2015. Nat. Hum. Behav. 2(9): 637-44.

Carlisle JB. 2012. The analysis of 168 randomised controlled trials to test data integrity. Anaesthesia 67(5): 521-37.

Carp, J. 2012. The secret lives of experiments: methods reporting in the fMRI literature." NeuroImage 63(1): 289-300.

Chalmers I, Glasziou P 2009. Avoidable waste in the production and reporting of research evidence. Lancet. 374(9683): 86-89.

Chambers CD. 2013. Registered Reports: A new publishing initiative at Cortex. Cortex. 49(3): 609-10.

Chambers CD, Mellor DT. 2018. Protocol transparency is vital for registered reports. Nat. Hum. Behav. 2: 791-792.

Chan, A-W, Hróbjartsson A, Haahr MT, Gøtzsche PC, Altman DG. 2004. Empirical evidence for selective reporting of outcomes in randomized trials: Comparison of protocols to published articles." JAMA. 291(20): 2457-65.

Chavalarias, D, Ioannidis JPA. 2010. Science mapping analysis characterizes 235 biases in biomedical research. J. Clin. Epidem. 63(11): 1205-15.

Chavalarias D, Wallach JD, Li AHT, Ioannidis JPA. 2016. Evolution of reporting p values in the biomedical literature, 1990-2015. JAMA. 315(11): 1141-48. 
Ciani O, Buyse M, Garside R, Pavey T, Stein K, Sterne JAC, Taylor RS. 2013. Comparison of treatment effect sizes associated with surrogate and final patient relevant outcomes in randomised controlled trials: Meta-epidemiological study. BMJ. 346: f457.

Cohen J. 1962. The statistical power of abnormal-social psychological research: a review. $J$. $A b$. Soc. Psychol. 65: 145-53.

Counsell A, Harlow LL. 2017. Reporting practices and use of quantitative methods in canadian journal articles in psychology." Canadian Psychology/Psychologie Canadienne. 58(2): 140-147.

Cramer AOJ, Ravenzwaaij D van, Matzke D, Steingroever H, Wetzels R, Grasman RPPP, Waldorp LJ, Wagenmakers E-J. 2016. Hidden multiplicity in exploratory multiway ANOVA: Prevalence and remedies. Psychon. Bul. Rev. 23(2): 640-47.

Cumming G, Fidler F, Leonard M, Kalinowski P, Christiansen A, Kleinig A, Lo J, McMenamin N, Wilson S. 2007. Statistical reform in psychology: is anything changing? Psychol. Sci. 18(3): 230-32.

Dalen HP van, Henkens K. 2012. Intended and unintended consequences of a publish-or-perish culture: a worldwide survey.” J. Am. Soc. Info. Sci. Tech. 63(7): 1282-93.

Dal-Ré, R, Ioannidis JPA, Bracken MB, Buffler PA, Chan A-W, Franco EL, La Vecchia C, Weiderpass E. 2014. Making prospective registration of observational research a reality. Sci. Trans. Med. 6(224): 224.

Dechartres A, Boutron I, Trinquart L, Charles P, Ravaud P. 2011. Single-center trials show larger treatment effects than multicenter trials: Evidence from a meta-epidemiologic study."Ann. Int. Med. 155(1): 39-51.

Dechartres A, Trinquart L, Atal I, Moher D, Dickersin K, Boutron I, ... Ravaud P. 2017. Evolution of poor reporting and inadequate methods over time in 20920 randomised controlled trials included in Cochrane reviews: research on research study. BMJ. 357: j2490.

Dickersin K, Rennie D. 2012. The evolution of trial registries and their use to assess the clinical trial enterprise. JAMA 307(17): 1861-64.

Dickersin K, Min YI, Meinert CL. 1992. Factors influencing publication of research results. Follow-up of applications submitted to two institutional review boards. JAMA 267(3): $374-78$. 
Dongen, NNN van, Doorn J van, Gronau QF, Ravenzwaaij D van, Hoekstra R, Haucke M, Lakens D, et al. 2019. Multiple perspectives on inference for two simple statistical scenarios. Am Stat. 73(S1): 328-339.

Dreber A, Pfeiffer T, Almenberg J, Isaksson S, Wilson B, Chen Y, Nosek BA, Johannesson M. 2015. Using prediction markets to estimate the reproducibility of scientific research. Proc. Nat. Acad. Sci. USA 112 (50): 15343-47.

Dwan K, Altman DG, Clarke M, Gamble C, Higgins JPT, Sterne JAC, Williamson PR, Kirkham JJ. 2014. Evidence for the selective reporting of analyses and discrepancies in clinical trials: a systematic review of cohort studies of clinical trials. PLOS Med. 11(6): e1001666.

Dwan K, Altman DG, Cresswell L, Blundell M, Gamble CL, Williamson PR. 2011. Comparison of protocols and registry entries to published reports for randomised controlled trials. Cochrane Database of Systematic Reviews , no. 1 (January): MR000031.

Dwan K, Gamble C, Williamson PR, Kirkham JJ, Reporting Bias Group. 2013. Systematic review of the empirical evidence of study publication bias and outcome reporting bias An updated review. PLOS ONE. 8(7): e66844.

Easterbrook PJ, Berlin JA, Gopalan R, Matthews DR. 1991. Publication bias in clinical research. Lancet 337(8746): 867-72.

Elms AC. 1975. The crisis of confidence in social psychology. Am. Psychol. 30(10): 967-976.

Ernst AF, Albers CJ. 2017. Regression assumptions in clinical psychology research practice-a systematic review of common misconceptions." PeerJ. 5: e3323.

Etz A, Vandekerckhove J. 2016. A Bayesian perspective on the Reproducibility Project: Psychology. PLOS One 11(2): e0149794.

Fanelli D. 2009. How many scientists fabricate and falsify research? A systematic review and meta-analysis of survey data. PloS One 4 (5): e5738.

Fanelli D. 2010. Do pressures to publish increase scientists' bias? An empirical support from US States Data. PLOS ONE. 4: e10271.

Fanelli D. 2011. "Negative results are disappearing from most disciplines and countries. Scientometrics 90(3): 891-904.

Fanelli D, Costas R, Ioannidis, JPA. 2017. Meta-assessment of bias in science. Proc. Nat. Acad. Sci. USA 114(14): 3714-19. 
Faust D, Meehl PE. 2002. Using meta-scientific studies to clarify or resolve questions in the philosophy and history of science. Phil. Sci. 69(S3): S185-S196.

Fidler F, Burgman MA, Cumming G, Buttrose R, Thomason N. 2006. Impact of criticism of null-hypothesis significance testing on statistical reporting practices in conservation biology." Conserv. Bio. 20(5): 1539-1544.

Fiedler K. 2011. Voodoo correlations are everywhere-not only in neuroscience. Perspect. Psychol. Sci. 6(2): 163-171.

Forsell E, Viganola D, Pfeiffer T, Almenberg J, Wilson B, Chen Y, Nosek BA, Johannesson M, Dreber A. 2018. Predicting replication outcomes in the Many Labs 2 study. J. Econ. Psych. In press. https://doi.org/10.1016/j.joep.2018.10.009.

Franco A, Malhotra N, Simonovits G. 2014. Publication bias in the social sciences: Unlocking the file drawer." Science 345(6203): 1502-5.

Franco A, Malhotra N, Simonovits G. 2016. Underreporting in psychology experiments. Social Psychological and Personality Science. 7(1): 8-12.

Franzoni C, Scellato G, Stephan P. 2011. Changing incentives to publish. Science 333(6043): $702-3$.

Fricker RD, Burke K, Han X, Woodall WH. 2019. Assessing the statistical analyses used in Basic and Applied Social Psychology after their p-value ban.” Am. Stat. 73(1): 374-384.

Gelman A, Loken E. 2014. The statistical crisis in science: Data-dependent analysis, a "garden of forking paths", explains why many statistically significant comparisons don't hold up. Am. Scient. 102: 460-465.

Gelman, A, Stern H. 2006. The difference between 'Significant' and 'Not Significant' is not itself statistically significant. Am. Stat. 60: 328-331.

Gigerenzer, G. 2004. Mindless statistics. J. Socio-Econ. 33: 587-606.

Giner-Sorolla R. 2012. Science or art? How aesthetic standards grease the way through the publication bottleneck but undermine science. Perspect. Psychol. Sci. 7(6):562-71.

Glasziou P, Altman DG, Bossuyt P, Boutron I, Clarke M, Julious S, Michie S, Moher D, Wager E. 2014. Reducing waste from incomplete or unusable reports of biomedical research. Lancet 383(9913): 267-76. 
Goldacre B, Drysdale H, Dale A, Milosevic I, Slade E, Hartley P, Marston C, Powell-Smith A, Heneghan C, Mahtani KR. 2019. COMPare: A prospective cohort study correcting and monitoring 58 misreported trials in real time. Trials. 20: 118.

Goodman SN. 1993. P values, hypothesis tests, and likelihood: implications for epidemiology of a neglected historical debate. Am. J. Epi. 137(5): 485-496.

Goodman SN. 2018. How sure are you of your result? Put a number on it. Nature. 564: 7.

Goodman SN. 2019. "Why is getting rid of p-values so hard? Musings on science and statistics. Am. Stat. 73(S1): 26-30.

Goodman SN, Fanelli D, Ioannidis JPA. 2016. What does research reproducibility mean? Sci. Trans. Med. 8(341): 1-6.

Gopal AD, Wallach JD, Aminawung JA, Gonsalves G, Dal-Ré R, Miller JE, Ross JS. 2018. Adherence to the International Committee of Medical Journal Editors' (ICMJE) prospective registration policy and implications for outcome integrity: a cross-sectional analysis of trials published in high-impact specialty society journals. Trials. 19(1): 448.

Grimes, DR, Bauch CT, Ioannidis JPA. 2018. Modelling science trustworthiness under publish or perish pressure. Roy. Soc. Open Sci. 5(1): 171511.

Hardwicke TE, Ioannidis JPA. 2018a. Mapping the universe of Registered Reports. Nat. Hum. Behav. 2: 793-79.

Hardwicke TE, Ioannidis JPA. 2018b. Populating the Data Ark: An attempt to retrieve, preserve, and liberate data from the most highly-cited psychology and psychiatry articles." PLOS One 13(8): e0201856.

Hardwicke TE, Mathur MB, MacDonald K, Nilsonne G, Banks GC, Kidwell MC, HofelichMohr A, et al. 2018. Data availability, reusability, and analytic reproducibility: Evaluating the impact of a mandatory open data policy at the journal Cognition.” Roy. Soc. Open Sci. 5(8): 180448.

Hardwicke TE, Frank MC, Vazire S, Goodman SN (in press). Should psychology journals adopt specialized statistical review? Adv. Meth. Prac. Psychol. Sci.

Hardwicke TE, Wallach JD, Kidwell MC, Ioannidis, JPA (2019). An empirical assessment of transparency and reproducibility-related research practices in the social sciences (20142017). MetaArXiv Preprints. Retrieved from: https://doi.org/10.31222/osf.io/6uhg5 
Hemkens LG, Contopoulos-Ioannidis DG, Ioannidis JPA. 2016. Agreement of treatment effects for mortality from routinely collected data and subsequent randomized trials: Metaepidemiological survey. BMJ 352.

Higginson AD, Munafò MR. 2016. Current incentives for scientists lead to underpowered studies with erroneous conclusions. PLOS Bio. 14(11): e2000995.

Hoekstra R, Finch S, Kiers HAL, Johnson A. 2006. Probability as certainty: dichotomous thinking and the misuse of p values. Psychon. Bul. Rev. 13(6): 1033-37.

IntHout J, Ioannidis JPA, Borm GF, Goeman JJ. 2015. Small studies are more heterogeneous than large ones: A meta-meta-analysis. J. Clin. Epi. 68(8): 860-69.

Ioannidis JPA 2005. Why most published research findings are false. PLOS Med. 2(8): e124.

Ioannidis JPA 2008. Why most discovered true associations are inflated. Epidemiology, 19(5): 640-48.

Ioannidis JPA 2012. Why science is not necessarily self-correcting. Perspect. Psychol. Sci. 7(6): 645-654.

Ioannidis JPA. 2014. How to make more published research true.” PLOS Med. 11(10): e1001747.

Ioannidis JPA. 2015. Handling the fragile vase of scientific practices. Addiction. 110(1): 9-10.

Ioannidis JPA 2016. The mass production of redundant, misleading, and conflicted systematic reviews and meta-analyses. Mil. Quart. 94(3): 485-514.

Ioannidis JPA 2017. The reproducibility wars: successful, unsuccessful, uninterpretable, exact, conceptual, triangulated, contested replication. Clin. Chem. 63(5): 943-45.

Ioannidis JPA. 2018. Meta-research: why research on research matters. PLOS Bio. 16(3): e2005468.

Ioannidis JPA. 2019. Retiring statistical significance would give bias a free pass. Nature. 567: 461.

Ioannidis JPA, Stanley TD, Doucouliagos H. 2017a. The power of bias in economics research. Econ. J. 127(605): F236-F265.

Ioannidis JPA, Caplan AL, Dal-Ré R. 2017b. Outcome reporting bias in clinical trials: Why monitoring matters.” BMJ. 356: j408.

Ioannidis JPA, Fanelli D, Dunne DD, Goodman SN. 2015. Meta-research: evaluation and improvement of research methods and practices. PLOS Bio. 13(10): e1002264. 
Ioannidis JPA, Greenland S, Hlatky MA, Khoury MJ, Macleod MR, Moher D, Schulz KF, Tibshirani R. 2014. Increasing value and reducing waste in research design, conduct, and snalysis. Lancet. 383 (9912): 166-75.

Ioannidis JPA, Trikalinos, TA. 2007. An exploratory test for an excess of significant findings. Clin. Trials. 4(3):245-53.

Iqbal SA, Wallach JD, Khoury MJ, Schully SD, Ioannidis JPA. 2016. Reproducible research practices and transparency across the biomedical literature. PLOS Bio. 14(1): e1002333.

John LK, Loewenstein G, Prelec D. 2012. Measuring the prevalence of questionable research practices with incentives for truth telling. Psycholo. Sci. 23(5): 524-32.

Jones CW, Keil LG, Holland WC, Caughey MC, Platts-Mills TF. 2015. Comparison of registered and published outcomes in randomized controlled trials: a systematic review. BMC Med. 13: 282.

Justice AC, Cho MK, Winker MA, Berlin JA, Rennie D. 1998. Does masking author identity improve peer review quality? A randomized controlled trial. PEER Investigators. JAMA. 280(3): 240-242.

Kaplan RM, Irvin VL. 2015. Likelihood of null effects of large NHLBI clinical trials has increased over time. PLOS One. 10(8): e0132382.

Kerr NL. 1998. HARKing: Hypothesizing after the Results Are Known. Pers. Soc. Psychol. Rev. 2(3): 196-217.

Kimmelman, J, Mogil JS, Dirnagl U. 2014. Distinguishing between exploratory and confirmatory preclinical research will improve translation. PLOS Bio. 12(5): e1001863.

Klein, O, Hardwicke TE, Aust F, Breuer J, Danielsson H, Hofelich-Mohr A, Ijzerman H, Nilsonne G, Vanpaemel W, Frank MC. 2018. A practical guide for transparency in psychological science. Collab. Psychol. 4(1): 20.

Klein RA, Vianello M, Hasselman F, Adams BG, Adams RB, Alper S, Aveyard M, et al. 2018. Many Labs 2: Investigating variation in replicability across samples and settings. $A d v$. Meth. Pract. Psychol. Sci. 1(4): 443-90.

Lane DM, Sándor A. 2009. Designing better graphs by including distributional Information and integrating words, numbers, and images. Psychol. Meth. 14(3): 239-57.

Lash TL, Vandenbroucke JP. 2012. Should preregistration of epidemiologic study protocols become compulsory? Epidemiology. 23(2): 184-88. 
Lazic SE, Clarke-Williams CJ, Munafò MR. 2018. What exactly is ' $N$ ' in cell culture and animal experiments? PLOS Bio. 16(4): e2005282.

Leamer EE. 1983. Let's take the con out of econometrics. Am. Econ. Rev. 73(1): 31-43.

Liddell T, Kruschke JK. 2018. Analyzing ordinal data with metric models: what could possibly go wrong?” J. Exp. Soc. Psychol. 79: 328-348.

Loder E, Loder S, Cook S. 2018. Characteristics and publication fate of unregistered and retrospectively registered clinical trials submitted to the BMJ over 4 Years." BMJ Open. 8(2): e020037.

Madigan D, Stang PE, Berlin JA, Schuemie M, Overhage JM, Suchard MA, Dumouchel B, Hartzema AG, Ryan PB. 2014. A systematic statistical approach to evaluating evidence from observational studies. Ann. Rev. Stat. App. 1: 11-39.

Makel MC, Plucker JA, Hegarty B. 2012. Replications in psychology research. Perspect. Psychol. Sci. 7: 537-542.

Marwick B, Boettiger C, Mullen L. 2017. Packaging data analytical work reproducibly using R (and friends). Am. Stat. 72(1): 80-88.

Mathieu S, Boutron I, Moher D, Altman DG, Ravaud P. 2009. Comparison of registered and published primary outcomes in randomized controlled trials. JAMA. 302(9): 977-84.

Matzke D, Nieuwenhuis S, Rijn H van, Slagter HA, van der Molen MW, Wagenmakers EJ. 2015. The effect of horizontal eye movements on free recall: A preregistered adversarial collaboration. J. Exp. Psychol. Gen.l 144(1): e1-15.

Mayo DG. 2018. Statistical Inference as Severe Testing: How to Get Beyond the Statistics Wars. Cambridge University Press.

McGillivray B, De Ranieri E. 2018. Uptake and outcome of manuscripts in Nature journals by review model and author characteristics. Res. Int. Peer Rev. 3: 5.

McShane BB, Gal D, Gelman A, Robert C, Tackett JL. 2019. Abandon statistical significance. Am. Stat. 73(1): 235-245.

Merton RK. 1973. The Sociology of Science: Theoretical and Empirical Investigations. University of Chicago Press.

Meyer MN. 2018. Practical tips for ethical data sharing. Adv. Meth. Prac. Psychol. Sci. 1(1): $131-144$. 
Miguel E, Camerer C, Casey K, Cohen J, Esterling KM, Gerber A, Glennerster R, et al. 2014. Promoting transparency in social science research. Science. 343(6166): 30-31.

Moher D, Dulberg CS, Wells GA. 1994. Statistical power, sample size, and their reporting in randomized controlled trials. JAMA. 272(2): 122-124.

Moher D, Jones A, Lepage L, and CONSORT Group (Consolidated Standards for Reporting of Trials). 2001. Use of the CONSORT statement and quality of reports of randomized trials: a comparative before-and-after evaluation. JAMA. 285(15): 1992-95.

Moher D, Naudet F, Cristea IA, Miedema F, Ioannidis JPA, Goodman SN. 2018. Assessing scientists for hiring, promotion, and tenure. PLOS Bio. 16(3): e2004089.

Morey RD., Chambers CD, Etchells PJ, Harris CR, Hoekstra R, Lakens D, Lewandowsky S, et al. 2016. The Peer Reviewers' Openness Initiative: incentivizing open research practices through peer review. Roy. Society Open Sci. 3(1): 150547.

Moshontz H, Campbell L, Ebersole CR, Ijzerman H, Urry HL, Forscher, PS, Grahe JE, et al. 2018. The Psychological Science Accelerator: advancing psychology through a distributed collaborative network. Adv. Meth. Pract. Psychol. Sci. 1(4): 501-15.

Mulkay MJ. 1976. Norms and ideology in science. Soc. Sci. Info. 15(4-5): 637-656.

Munafò MR, Nosek BA, Bishop BVM, Button KS, Chambers CD, Percie du Sert N, Simonsohn U, Wagenmakers EJ, Ware JJ, Ioannidis JPA. 2017. A manifesto for reproducible science. Nat. Hum. Behav. 1(1): 0021.

Murad, MH, Wang Z. 2017. Guidelines for reporting meta-epidemiological methodology research. Evi-Bas Med 22(4): 139-42.

Naudet F, Sakarovitch C, Janiaud P, Cristea IA, Fanelli D, Moher D, Ioannidis JPA. 2018. Data sharing and reanalysis of randomized controlled trials in leading biomedical journals with a full data sharing policy: survey of studies published in The BMJ and PLOS Medicine. BMJ. 360: k400.

Nelson LD, Simmons J, Simonsohn U. 2018. Psychology’s renaissance. Ann. Rev. Psychol. 69(1): 511-34.

Nieuwenhuis S, Forstmann BU, Wagenmakers E-J. 2011. Erroneous analyses of interactions in neuroscience: a problem of dignificance. Nat. Neurosci. 14(9): 1105-7.

Nosek BA, Alter G, Banks GC, Borsboom D, Bowman SD, Breckler SJ, Buck S, et al. 2015. Promoting an open research culture. Science. 348(6242): 1422-25. 
Nosek BA, Ebersole CR, DeHaven AC, Mellor DT. 2018. The preregistration revolution. Proc. Nat. Acad. Sci. USA 115(11): 2600-2606.

Nosek BA, Errington TM. 2017. Making sense of replications. eLife. 6: e23383.

Nosek BA, Spies JR, Motyl M. 2012. Scientific utopia: II. Restructuring incentives and practices to promote truth over publishability. Perspect. Psycholog. Sci. 7(6): 615-31.

Nuijten MB, Borghuis J, Veldkamp CLS, Dominguez-Alvarez L, Assen MALM van, Wicherts JM. 2017. Journal data sharing policies and statistical reporting inconsistencies in psychology. Collab. Psychol. 3(1): 31.

Nuijten MB, Hartgerink CHJ, Assen MALM van, Epskamp S, Wicherts JM. 2016. The prevalence of statistical reporting errors in psychology (1985-2013)." Behav. Res. Meth. 48(4): 1205-26.

O’Boyle EH, Banks GC, Gonzalez-Mulé E. 2013. The Chrysalis Effect: how ugly data metamorphosize into beautiful articles. Acad. Man. Proc. 43(2): 376-399.

Open Science Collaboration. 2015. Estimating the reproducibility of psychological science. Science. 349(6251): aac4716.

Orben A, Przybylski AK. 2019. The association between adolescent well-being and digital technology use. Nat. Hum. Behav. 3: 173-182.

Page MJ, Higgins JPT, Clayton G, Sterne JAC, Hróbjartsson A, Savović J. 2016. Empirical evidence of study design biases in randomized trials: Systematic review of metaepidemiological studies. PLOS One 11(7): e0159267.

Pashler H, Wagenmakers EJ. 2012. Editors' introduction to the special section on replicability in psychological science: a crisis of confidence?” Perspect. Psycholog. Sci. 7(6): 528-30.

Patel CJ. Burford B, Ioannidis JPA. 2015. Assessment of vibration of effects due to model specification can demonstrate the instability of observational associations. J. Clin. Epi. 68(9): 1046-58.

Pereira TV, Horwitz RI, Ioannidis JPA. 2012. Empirical evaluation of very large treatment effects of medical interventions." JAMA. 308(16): 1676-84.

Phillips CV. 2004. Publication bias in situ. BMC Med. Res. Meth. 4: 20.

Poldrack RA, Baker CI, Durnez J, Gorgolewski KJ, Matthews PM, Munafò MR, Nichols TE, Poline J-B, Vul E, Yarkoni T. 2017. Scanning the horizon: towards transparent and reproducible neuroimaging research.” Nat. Rev. Neurosci. 18(2): 115-26. 
Prinz F, Schlange T. Asadullah K. 2011. Believe it or not: how much can we rely on published data on potential drug targets?" Nat. Rev. Drug Discov. 10(9): 712.

Robinson KA, Goodman SN. 2011. A systematic examination of the citation of prior research in reports of randomized, controlled trials. Ann. Int. Med. 154(1): 50-55.

Rosenthal R. 1966. Experimenter Effects in Behavioral Research. CT, US: Appleton-CenturyCrofts.

Rosenthal R. 1979. The file drawer problem and tolerance for null results. Psychol. Bul. 86(3): 638-41.

Ross JS, Tse T, Zarin DA, Xu H, Zhou L, \& Krumholz HM. 2012. Publication of NIH funded trials registered in ClinicalTrials.gov: cross sectional analysis. BMJ.344: d7292.

Ross-Hellauer T, Görögh E. 2019. Guidelines for open peer review implementation. Res. Int. Peer Rev. 4: 4.

Rowhani-Farid, A., Barnett AG. 2016. Has open data arrived at the British Medical Journal (BMJ)? An observational study. BMJ Open 6(10): e011784.

Schatz P, Jay KA, McComb J, McLaughlin JR. 2005. Misuse of statistical tests in archives of clinical neuropsychology publications. Arch. Clin. Neuropsych. 20(8): 1053-59.

Scott A, Rucklidge JJ, Mulder RT. 2015. Is mandatory prospective trial registration working to prevent publication of unregistered trials and selective outcome reporting? An observational study of five psychiatry journals that mandate prospective clinical trial registration.” PLOS One 10(8): e0133718.

Sedlmeier P, Gigerenzer G. 1989. Do studies of statistical power have an effect on the power of studies? Psychol. Bul. 105(2): 309-16.

Seminara D, Khoury MJ, O’Brien TR, Manolio T, Gwinn ML, Little J, Higgins JPT, et al. 2007. The emergence of networks in human genome epidemiology: challenges and opportunities. Epidemiology. 18(1): 1-8.

Schuemie MJ, Ryan PB, DuMouchel W, Suchard MA, Madigan D. 2014. Interpreting observational studies: why empirical calibration is needed to correct p-values. Stat. Med. 33(2): 209-218.

Shamseer L, Hopewell S, Altman DG, Moher D, Schulz KF. 2016. Update on the endorsement of CONSORT by high impact factor journals: a survey of journal 'Instructions to Authors' in 2014. Trials. 17(1): 301. 
Silberzahn R, Uhlmann EL, Martin DP, Anselmi P, Aust F, Awtrey E, Bahník S, et al. 2018. Many analysts, one data set: making transparent how variations in analytic choices affect results. Adv. Meth. Prac. Psychol. Sci. 1(3): 337-56.

Simmons JP, Nelson LD, Simonsohn, U. 2011. False-positive psychology: undisclosed flexibility in data collection and analysis allows presenting anything as significant. Psychol. Sci. 22(11): 1359-66.

Simonsohn, U. 2013. Just post it: the lesson from two cases of fabricated data detected by statistics alone. Psychol. Sci. 24(10): 1875-88.

Smaldino PE, McElreath R. 2016. The natural selection of bad science. Roy. Soc. Open Sci. 3(9): 160384.

Stanley DJ, Spence JR. 2014. Expectations for replications: Are yours realistic? Perspect. Psychol. Sci. 9(3): 305-18.

Steegen, S, Tuerlinckx F, Gelman A, Vanpaemel, W. 2016. Increasing transparency through a multiverse analysis." Perspect. Psychol. Sci. 11(5): 702-12.

Sterling TD. 1959. Publication decisions and their possible effects on inferences drawn from tests of significance — or vice versa. J. Am. Stat. Assoc. 54(285): 30-34.

Stodden V, Seiler J, Ma Z. 2018. An empirical analysis of journal policy effectiveness for computational reproducibility. Proc. Nat. Acad. Sci. USA 115(11): 2584-89.

Strasak AM, Zaman Q, Marinell G, Pfeiffer KP, Ulmer H. 2007. The use of statistics in medical research. Am. Stat. 61(1): 47-55.

Spellman BA. 2015. A short (personal) future history of Revolution 2.0. Pers. Psych. Sci.10(6): 886-899.

Szucs D, Ioannidis JPA. 2017a. Empirical assessment of published effect sizes and power in the recent cognitive neuroscience and psychology literature. PLOS Bio. 15(3): e2000797.

Szucs D, Ioannidis JPA. 2017b. When null hypothesis significance testing is unsuitable for research: A reassessment. Front. Hum. Neurosci. 11:390.

Tierney JF, Vale C, Riley R, Smith CT, Stewart L, Clarke M, Rovers M. 2015. Individual participant data (IPD) meta-analyses of randomised controlled trials: guidance on their use." PLOS Med. 12(7): e1001855.

Trinquart, L, Dunn AG, Bourgeois FT. 2018. Registration of published randomized trials: a systematic review and meta-analysis. BMC Med. 16(1): 173. 
Tsakiris M, Martin R, Wagemans J. 2018. Re-thinking Cognition's open data policy: responding to Hardwicke and colleagues' evaluation of its impact. Cognition, October.

Turner L, Shamseer L, Altman DG, Schulz KF, Moher D. 2012. Does use of the CONSORT Statement impact the completeness of reporting of randomised controlled trials published in medical journals? A Cochrane review. Syst. Rev. 29:

Vanpaemel W, Vermorgen M, Deriemaecker L, Storms G. 2015. Are we wasting a good crisis? The availability of psychological research data after the storm. Collabra. 1(1): 1-5. Vasilevsky NA, Brush MH, Paddock H, Ponting L, Tripathy SJ, Larocca GM, Haendel MA. 2013. On the reproducibility of science: unique identification of research resources in the biomedical literature. PeerJ 1:e148.

Vazire S. 2017. Quality uncertainty erodes trust in science. Collab. Psychol. 13(4): 411-417.

Vines TH, Albert AYK, Andrew RL, Débarre F, Bock DG, Franklin MT, Gilbert KJ, Moore J-S, Renaut S, Rennison DJ. 2014. The availability of research data declines rapidly with article age. Cur. Bio. 24(1): 94-97.

Voytek B. 2016. The virtuous cycle of a data ecosystem. PLOS Comput. Biol. 12(8): e1005037.

Vul E, Harris C, Winkielman P, Pashler H. 2009. Puzzlingly high correlations in fMRI Studies of emotion, personality, and social cognition.” Perspect. Psychol. Sci. 4(3): 274-90.

Wagenmakers, E-J. 2007. A practical solution to the pervasive problems of p values. Psychon. Bul. Rev. 14(5): 779-804.

Wagenmakers E-J, Wetzels R, Borsboom D, van der Maas JLJ, Kievit RA. 2012. An agenda for purely confirmatory research.” Perspect. Psychol. Sci. 7(6): 632-38.

Walker RL, Sykes L, Hemmelgarn BR, Quan H. 2010. Authors' opinions on publication in relation to annual performance assessment." BMC Med. Ed. 10: 21.

Wallach JD, Boyack KW, Ioannidis JPA. 2018. Reproducible research practices, transparency, and open access data in the biomedical literature, 2015-2017. PLOS Bio. 16(11): e2006930.

Wallach, JD, Sullivan PG, Trepanowski JF, Sainani KL, Steyerberg EW, Ioannidis JPA. 2017. Evaluation of evidence of statistical support and corroboration of subgroup claims in randomized clinical trials." JAMA Int. Med. 177(4): 554-60.

Wasserstein RL, Schirm AL, Lazar NA. 2019. Moving to a world beyond 'P < 0.05."' Am. Stat. 73(S1): 1-19. 
Weissgerber TL, Garcia-Valencia O, Garovic VD, Milic NM, Winham SJ. 2018. Why we need to report more than 'data were analyzed by t-tests or ANOVA.'” eLife 7: e36163.

Weissgerber TL, Milic NM, Winham SJ, Garovic VD. 2015. Beyond bar and line graphs: time for a new data presentation paradigm. PLOS Bio. 13(4): e1002128.

Wicherts JM, Borsboom D, Kats J, Molenaar D. 2006. The poor availability of psychological research data for reanalysis. Am. Psychol. 61(7): 726-28.

Wiseman R, Watt C, Kornbrot D. 2019. Registered Reports: An early example and analysis. PeerJ. 7: e6232.

World Medical Association. 2013. "World Medical Association Declaration of Helsinki: ethical principles for medical research involving human subjects.” JAMA. 310(20): 2191-94.

Wu L, Wang D, Evans JA. 2019. Large teams develop and small teams disrupt science and technology. Nature. 566(7744): 378-82.

Yong, E. 2012. Replication studies: bad copy. Nature 485(7398): 298-300.

Young NS, Ioannidis JPA, Al-Ubaydli O. 2008. Why current publication practices may distort science. PLOS Med. 5(10): e201.

Zarin DA, Tse T, Ide. C. 2005. Trial registration at ClinicalTrials.gov between May and October 2005. New Eng. J. Med. 353(26): 2779-87.

Zarin DA, Tse T, Williams RJ, Califf RM, Ide NC. 2011. The ClinicalTrials.gov results database--update and key issues. New Eng. J. Med. 364(9): 852-60. 